\title{
Thermodynamics of Fcc Al Crystal from First Principles - Performance of Local Density and Generalized Gradient Approximations
}

\author{
P. Scharoch*, J. Peisert and K. Tatarczyk \\ Institute of Physics, Wrocław University of Technology \\ Wyb. Wyspiańskiego 27, 50-370 Wrocław, Poland
}

(Received August 2, 2007)

\begin{abstract}
Phonon dispersion relations in fcc $\mathrm{Al}$ crystal were calculated from first principles using density functional perturbation theory, as implemented in ABINIT code. The results are compared with experimental data as well as with the results of previously done $a b$ initio calculations based on the direct method. A slightly better agreement of density functional perturbation theory phonons with experiment can be observed. The $a b$ initio phonon energies were used to evaluate the partition function of the crystal, using the Monkhorst-Pack integration scheme. The quasiharmonic approximation was applied to relate the temperature dependent part of the free energy to volume. The lattice constant dependence of phonon energies was found to be almost linear, so the second order polynomial was considered as sufficient to approximate the dependence. A few examples of thermodynamic characteristics were evaluated: isobaric specific heat, linear thermal expansion coefficient, isothermal bulk modulus, and compared with the experimental data. The calculation was done both in the local density and the generalized gradient approximations for the exchange-correlation energy. The agreement with the experimental data appears to be very satisfactory, although better in the local density approximation than in the generalized gradient approximation.
\end{abstract}

PACS numbers: 65.40.--b, 63.20.Dj

\section{Introduction}

The computational methods based on the density functional theory (DFT), developing quickly over the last decades, provide very promising opportunities to

*corresponding author; e-mail: Pawel.Scharoch@pwr.wroc.pl 
study various aspects of physics of polyatomic systems from first principles, i.e. using only fundamental theories and universal constants.

This paper deals with a particular topic: thermal properties of crystals. The approach is based on the quasiharmonic approximation. Several papers have appeared so far in which the $a b$ initio calculations of crystal thermodynamics have been reported (e.g. [1-4]). The computational procedure involves the evaluation of the total energy and phonon energies, which are then used to calculate the phonon partition function, the fundamental quantity in statistical thermodynamics. The phonon energies can be calculated from first principles either using the direct method (e.g. [5]) or with the use of the density functional perturbation theory (DFPT) [6-12]. In this work we use the DFPT method, as implemented in the ABINIT code [13]. The phonon dispersion relations, calculated both for the local density approximation (LDA) (Ceperley and Alder, and Perdew and Zunger $[14,15])$ and generalized gradient approximation (GGA) (Perdew et al. [16]), are compared with experimental data and with the results of the direct method. On that basis the phonon partition function is evaluated which then serves to develop the whole thermodynamics. Some examples of thermodynamic characteristics are shown and compared with experiment. The results obtained allow us to compare the performance of commonly used approximations for the exchange-correlation energy, LDA and GGA.

In the following two sections we summarize briefly the theoretical background and then present examples of results, including phonon dispersion relations and some chosen thermodynamic characteristics.

\section{Method}

The thermodynamic analysis is performed within a concept of canonical ensemble. The following notation is introduced [17]: $X_{i}=\exp \left(-\beta E_{i}\right)$, where the phonon energy $E_{i}=\hbar \omega_{i}$ and $\omega_{i}$ is the frequency of the normal mode of index $i$, $\beta=1 / k_{\mathrm{B}} T, T$ is the temperature of the system and $k_{\mathrm{B}}$ - the Boltzmann constant. The single mode quantities: partition function $Z_{i}$, free energy $F_{i}$, internal energy $U_{i}$, entropy $S_{i}$, and specific heat $C_{i}$ assume the well-known forms

$$
\begin{aligned}
Z_{i} & =\frac{\sqrt{X_{i}}}{1-X_{i}}, \\
F_{i} & =-\frac{1}{\beta} \ln \left(Z_{i}\right), \\
U_{i} & =\frac{E_{i}}{2} \frac{1+X_{i}}{1-X_{i}}, \\
S_{i} & =\frac{U_{i}-F_{i}}{T},
\end{aligned}
$$




$$
C_{i}=k_{\mathrm{B}} \beta^{2} E_{i}{ }^{2} \frac{X_{i}}{\left(1-X_{i}\right)^{2}},
$$

and the respective quantities for the whole system

$$
\begin{aligned}
& Z=\prod_{i} Z_{i}, \\
& F=\sum_{i} F_{i}+E_{0}^{\mathrm{tot},} \\
& U=\sum_{i} U_{i}+E_{0}^{\mathrm{tot}}, \\
& S=\sum_{i} S_{i}, \\
& C_{V}=\sum_{i} C_{i},
\end{aligned}
$$

where $E_{0}^{\text {tot }}$ is the total energy of the system at static equilibrium and the summation (and product) runs over all normal modes.

In the approach presented above the temperature dependent part of the free energy does not contain volume, so in the equation of state

$$
P=-\left(\frac{\partial F}{\partial V}\right)_{T}
$$

with $V$ denoting volume, the pressure $P$ is related to volume only via the volume dependence of static total energy $E^{\text {tot }}(V)$. This means that pressure and consequently volume would not depend on temperature. The situation changes if the mode frequencies are related to volume (the quasiharmonic approximation). Below a brief summary of the model is presented.

Staying within the harmonic approximation, we take into account the fact that phonon frequencies depend on volume, $\omega_{i}=\omega_{i}(V)$. This effect can be clearly observed in ab initio calculations: even small variation of the lattice constant (on the order of $1 \%$ ) results in substantial changes in phonon energies (up to a few percent). As a measure of these changes, it is a common practice to introduce a dimensionless quantity called the Grüneisen number, defined as follows:

$$
\gamma_{i}=-\frac{\mathrm{d} \ln \left(\omega_{i}\right)}{\mathrm{d} \ln (V)} .
$$

The Grüneisen number depends on normal mode, therefore must be indexed by $i$. One can derive now the expressions for various thermodynamic quantities, from standard definitions. The formulae (6)-(10) remain unchanged (except for the fact that all the quantities now depend on crystal volume). A few examples of expressions for other functions are shown below.

- The equation of state (Eq. (11)) takes the form (the Mie-Grüneisen equation of state): 


$$
P(V, T)=P_{0}(V)+\frac{1}{V} \sum_{i} U_{i} \gamma_{i},
$$

where $P_{0}$ is the static contribution to pressure, equal to $-\partial E^{\text {tot }}(V) / \partial V$. In this formula and all the formulae below the summation runs over all normal modes.

- The isothermal modulus of elasticity

$$
\begin{aligned}
B_{T} & =-V\left(\frac{\partial P}{\partial V}\right)_{T}=B_{T 0}+\left(P-P_{0}\right)+\frac{1}{V} \sum_{i} \gamma_{i}^{2}\left(U_{i}-C_{i} T\right) \\
& -\sum_{i} U_{i} \frac{\partial \gamma_{i}}{\partial V}
\end{aligned}
$$

where $B_{T 0}$ is a respective static contribution.

- The isobaric (at constant pressure) volume thermal expansion coefficient

$$
A_{P}=\frac{1}{V}\left(\frac{\partial V}{\partial T}\right)_{P}=\frac{1}{V B_{T}} \sum_{i} \gamma_{i} C_{i}
$$

- The isobaric (at constant pressure) heat capacity

$$
C_{P}=C_{V}+A_{P}^{2} B_{T} V T \text {. }
$$

- The adiabatic (isoentropic) modulus of elasticity

$$
B_{V}=B_{T} \frac{C_{P}}{C_{V}}
$$

In the quantities above the static total energy as a function of volume, $E^{\text {tot }}(V)$ (together with its first and the second derivatives) appears. In principle it can be scanned directly in $a b$ initio calculations. There are, however, analytic expressions for this function which can be used to fit the ab initio data. Here, the very popular one, given by the Murnaghan equation of state [18], has been chosen

$$
E^{\mathrm{tot}}(V)-E^{\mathrm{tot}}\left(V_{0}\right)=\frac{B_{0} V}{B_{0}^{\prime}}\left[\frac{\left(V_{0} / V\right)^{B_{0}^{\prime}}}{B_{0}^{\prime}-1}+1\right]-\frac{B_{0} V_{0}}{B_{0}^{\prime}-1},
$$

where $V_{0}, B_{0}, B_{0}^{\prime}$ are the parameters corresponding to the static equilibrium geometry and they denote respectively: volume, modulus of elasticity, and its derivative with respect to pressure. With this parameterization the static contribution to pressure takes the form

$$
P_{0}(V)=\frac{B_{0}}{B_{0}^{\prime}}\left[\left(\frac{V_{0}}{V}\right)^{B_{0}^{\prime}}-1\right]
$$

and the static contribution to bulk modulus $\left(B_{T 0}\right)$ reads

$$
B_{T 0}(V)=B_{0}\left(\frac{V_{0}}{V}\right)^{B_{0}^{\prime}} \text {. }
$$

In Fig. 1 the graphs of the total energy vs. lattice constants (Murnaghan curves fitted to ab initio data) are shown both for LDA and GGA. 

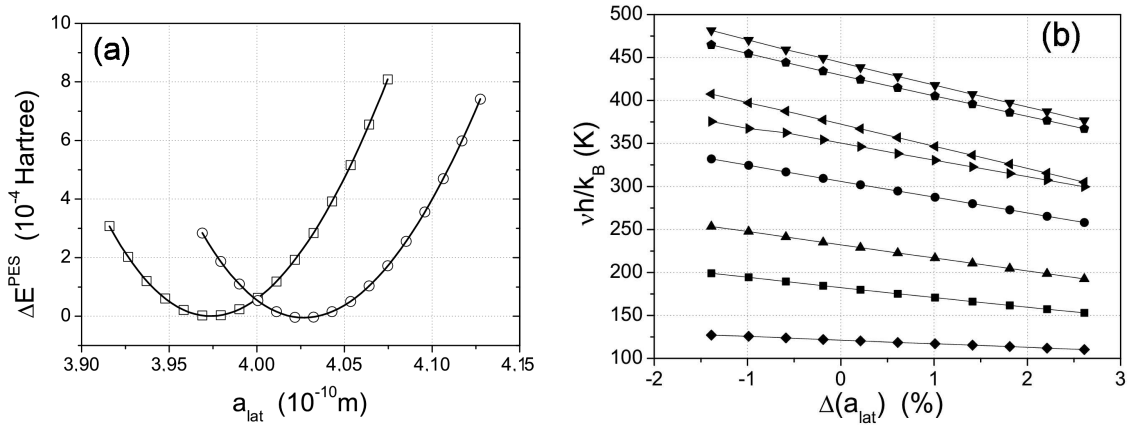

Fig. 1. (a) The potential energy surface (the total energy variation with the lattice constant as a variable); squares — LDA, circles — GGA, solid lines — the Murnaghan equation [18] fit to ab initio data. (b) Phonon energies at a few randomly chosen wave vectors in the Brillouin zone as functions of the lattice constant deviation from its static equilibrium value (LDA only). In the large range of deviations (2.5\% corresponds to the melting point at normal pressure) the dependence looks almost linear which justifies the second order polynomial approximation. One may note the general tendency: the bigger is the phonon energy the bigger is the decrease rate.

The choice of the model function for the phonon energy vs. volume dependence is also an important point. A common practice is to use the power law or the Taylor expansion of phonon energy versus the deviation of volume from equilibrium, either linear or up to the second order term (e.g. [1]). In this work it has been shown that the lattice constant dependence of phonon energies is almost linear (see Fig. 1), so it has been decided to approximate the dependence with the second order polynomial.

\section{Results of calculations}

\subsection{Computational details}

The results presented in this paper have been obtained using the following approximations and computational parameters. Both the LDA $[14,15]$ and the GGA [16] have been exploited. The pseudopotentials have been generated with the use of FHI98PP code [19]. The non-local core-valence exchange-correlation (XC) interaction has been included. All the $a b$ initio calculations (the ground state and the linear response function) have been done with the use of the ABINIT [13] code. The cut-off energy of $E_{\text {cut }}=20$ Hartree has been applied and the Monkhorst-Pack [20] $k$-points mesh $(6,6,6)$ shifted by $(0.5,0.5,0.5),(0.5,0.0,0.0),(0.0,0.5,0.0)$, $(0.0,0.0,0.5)$. The same $k$-point mesh has been used in the calculation of the electronic ground state, the response function and in the calculation of the phonon partition function. The choice of all the parameters have been preceded by careful study of convergence. 


\subsection{Total energy and phonons}

Figure 1a presents the lattice constant dependence of the static total energy (potential energy surface, $E^{\mathrm{PES}}$ ) — the Murnaghan equation of state fitted to $a b$ initio data. The GGA curve is shifted towards a larger lattice constant relative to LDA, which is the known property of the approximations. The static lattice constants and bulk moduli are listed in Table. In the graph in Fig. 1b the phonon energies at a few randomly chosen $k$ points in the Brillouin zone (BZ) as functions of the lattice constant deviation from its static equilibrium value (LDA only) can be seen. In the large range of deviations (2.5\% corresponds to the melting point at normal pressure) the dependence looks almost linear which justifies the second order polynomial approximation. One may also note the general tendency: the bigger is the phonon energy the bigger is the decrease rate.

TABLE

The static lattice constants and bulk moduli obtained from the Murnaghan fit to ab initio data, compared with the experimental data.

\begin{tabular}{c|c|c|c|c}
\hline \hline & $a_{\text {lat }}[\AA]$ & $\Delta a_{\text {lat }}[\%]$ & $B_{0}[\mathrm{kbar}]$ & $\Delta B_{0}[\%]$ \\
\hline experiment & 4.049 & 0 & 760 & 0 \\
LDA & 3.974 & -1.8 & 848 & -11.6 \\
GGA & 4.027 & -0.5 & 779 & -2.5
\end{tabular}

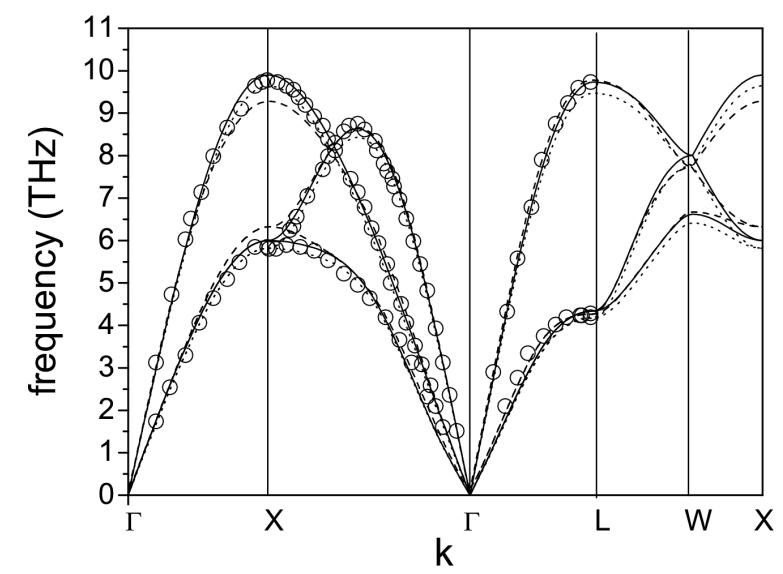

Fig. 2. Phonon dispersion relations along high symmetry directions in the BZ; circles - experimental points according to [21], solid/dotted line — DFPT results with LDA $[14,15] /$ GGA [16] for the XC functional, calculated with the use of the ABINIT code [13]; dashed line — direct method result [22].

In Fig. 2 the phonon dispersion relations for the equilibrium lattice constants are drawn. The first principles results, both, those based on the DFPT method 
(LDA and GGA) and those based on the direct method (LDA only) are compared with experimental data. The general observation is that all the ab initio results reproduce very well the measurement, however, in the case of the DFPT method the agreement is better (particularly in LDA), probably because in that method the range of interaction is not limited by the choice of supercell, as in the direct method.

\subsection{Thermodynamics}

In Figs. 3-5 some chosen thermodynamic characteristics calculated from first principles are presented and compared with experimental data. These are:
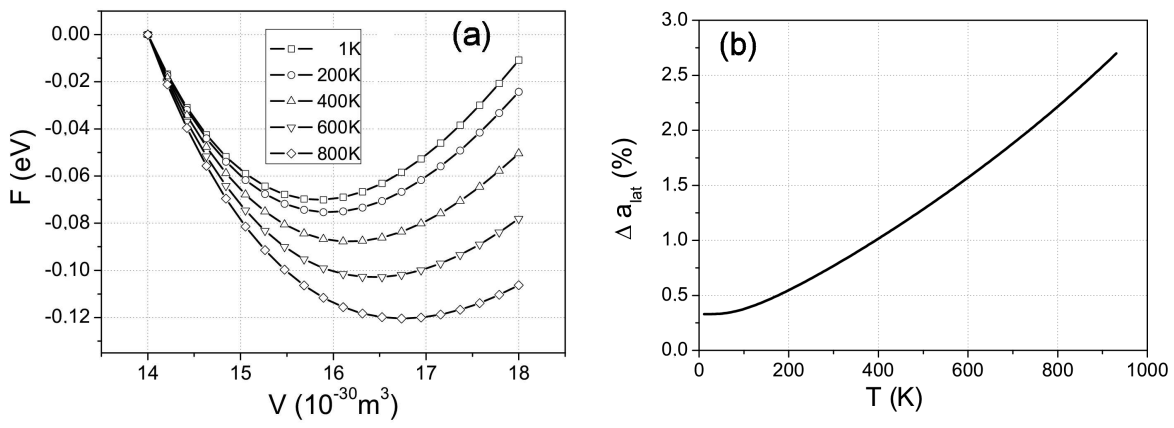

Fig. 3. (a) The free energy as a function of unit cell volume at different temperatures; points - $a b$ initio (LDA) data calculated within quasiharmonic approximation; the shift of the free energy minimum with temperature is responsible for the thermal expansion phenomenon (for clearer view all curves have been hooked at the same energy in their initial points). (b) The relative variation of the lattice constant as a function of temperature calculated $a b$ initio (LDA); the curve corresponds to the linear expansion coefficient shown in Fig. 4 and the shift of the free energy minimum shown in the graph in (a).
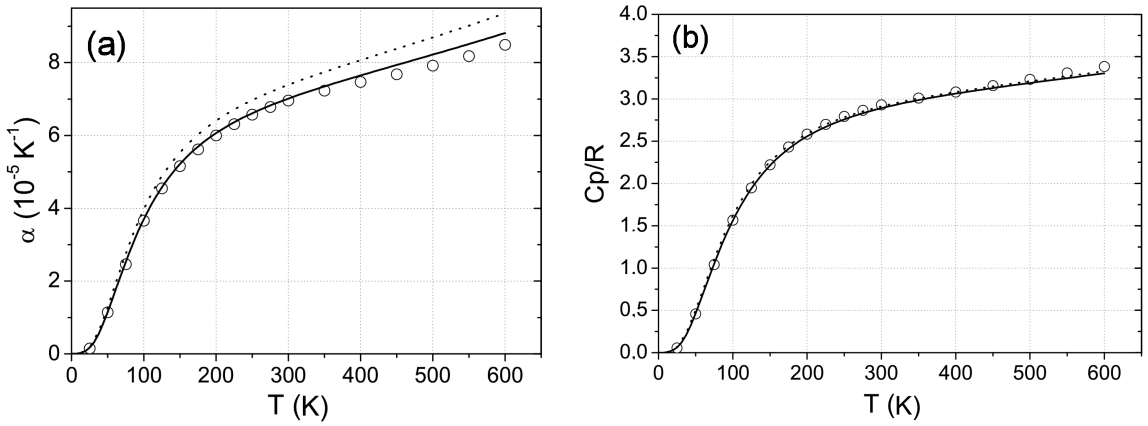

Fig. 4. The linear thermal expansion coefficient (a), and the isobaric specific heat (b), ab initio data vs. experiment; solid line - LDA, dotted line - GGA, circles - experimental data according to [23]. 

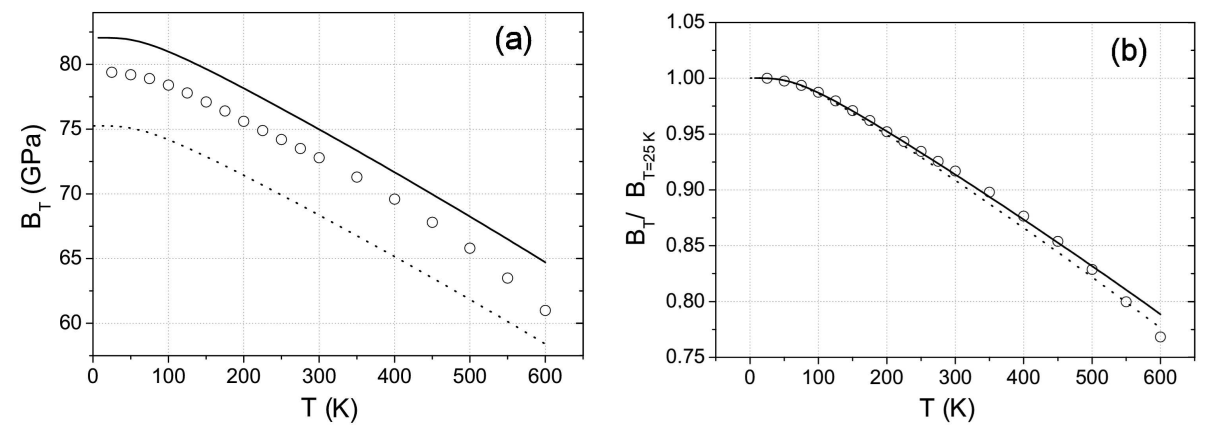

Fig. 5. The isothermal bulk modulus (a) and the isothermal bulk modulus normalized to the value at $25 \mathrm{~K}(\mathrm{~b})$, ab initio data vs. experiment; solid line — LDA, dotted line - GGA, circles - experimental data according to [23].

free energy as a function of the lattice constant (at a few temperatures), the linear thermal expansion coefficient, and the isothermal bulk modulus (modulus of compressibility). The agreement with experimental data is very satisfactory. The discrepancy between theoretical and experimental absolute value of the bulk modulus results from the static contribution (see Table). Although the GGA gives better values of the static lattice constant and the static bulk modulus, it seems that the thermal behavior is better reproduced by the LDA (see e.g. Fig. 4).

\section{Conclusions}

The following conclusions can be drawn from the presented results of $a b$ initio calculations:

- Ab initio calculations based on quasiharmonic approximation reproduce well thermodynamic characteristics of fcc Al crystal.

- The GGA provides better values of static parameters (lattice constant, bulk modulus) but LDA provides very good agreement with experiment of dynamics and thermodynamics of the fcc $\mathrm{Al}$ crystal

- The conclusions are in agreement with those reported in other theoretical works (e.g. [1, 24, 3, 4]).

- The thermal expansion phenomenon of fcc $\mathrm{Al}$ is the entropy driven effect, and the harmonic contribution to entropy is crucial.

The last point in the list above deserves special attention. The thermal expansion of the $\mathrm{Al}$ crystal (and probably many other crystals) is just the entropy effect and not the nonlinear one, as it is sometimes explained in the literature. Any "thermal shift" of atoms cannot take place since the single atom potential has an inversion symmetry, although it can be strongly anharmonic. The nonlinear contribution to entropy seems to be negligible at low temperatures but it might be responsible for the discrepancies between calculations and experiment observed 
at temperatures close to the melting point. The quasiharmonic approximation, which is usually thought of as based on the a priori assumption, gains then obvious justification: the thermal expansion of some crystals is connected with the fact that the entropy increases with increasing lattice constant because phonon energies decrease, and the static energy shift is compensated by the $S T$ term in the free energy (Fig. 3).

\section{Acknowledgments}

The calculations for this work were done in the Wrocław Center for Networking and Supercomputing.

\section{References}

[1] A. Debernardi, M. Alouani, H. Dreysse, Phys. Rev. B 63, 064305 (2001).

[2] J. Xie, S. de Gironcoli, S. Baroni, M. Scheffler, Phys. Rev. B 59, 965 (1999).

[3] A.A. Quong, A.Y. Liu, Phys. Rev. B 56, 7767 (1997).

[4] S. Biernacki, M. Scheffler, Phys. Rev. Lett. 63, 290 (1989).

[5] K. Parlinski, Y. Kawazoe, Phys. Rev. B 60, 15511 (1999).

[6] S. Baroni, P. Gianozzi, A. Testa, Phys. Rev. Lett. 58, 1861 (1987).

[7] X. Gonze, J.-P. Vigneron, Phys. Rev. B 39, 13120 (1989).

[8] X. Gonze, Phys. Rev. A 52, 1096 (1995).

[9] S. de Gironcoli, Phys. Rev. B 51, 6773 (1995).

[10] X. Gonze, Phys. Rev. B 55, 10337 (1997).

[11] X. Gonze, C. Lee, Phys. Rev. B 55, 10335 (1997).

[12] S. Baroni, S. de Gironcoli, A. Dal Corso, Rev. Mod. Phys. 73, 515 (2001).

[13] X. Gonze, J.-M. Beuken, R. Caracas, F. Detraux, M. Fuchs, G.-M. Rignanese, L. Sindic, M. Verstraete, G. Zerah, F. Jollet, M. Torrent, A. Roy, M. Mikami, Ph. Ghosez, J.-Y. Raty, D.C. Allan, Comput. Mater. Sci. 25, 478 (2002).

[14] D.M. Ceperley, B.J. Alder, Phys. Rev. Lett. 45, 567 (1980).

[15] J.P. Perdew, A. Zunger, Phys. Rev. B 23, 5048 (1981).

[16] J.P. Perdew, K. Burke, M. Ernzerhof, Phys. Rev. Lett. 77, 3865 (1996).

[17] P. Scharoch, Acta Phys. Pol. A 106, 487 (2004).

[18] F.D. Murnaghan, Proc. Natl. Acad. Sci. USA 30, 244 (1944).

[19] M. Fuchs, M. Scheffler, Comput. Phys. Commun. 119, 67 (1999).

[20] H.J. Monkhorst, J.D. Pack, Phys. Rev. B 13, 5188 (1976).

[21] R. Stedman, G. Nilson, Phys. Rev. 145, 492 (1966).

[22] P. Scharoch, K. Parlinski, A. Kiejna, Acta Phys. Pol. A 97, 349 (2000).

[23] D.C. Wallace, Thermodynamics of Crystals, Dover, New York 1972.

[24] J. Xie, S. de Gironcoli, S. Baroni, M. Scheffler, Phys. Rev. B 59, 970 (1999). 